\title{
Phonon confinement using spirally designed elastic resonators in discrete continuum
}

\author{
Sourav Banerjee*, Riaz Uddin Ahmed \\ Dept. of Mechanical Engineering, University of South Carolina, Columbia, South Carolina, USA
}

\section{Email address:}

banerjes@cec.sc.edu(S. Banerjee), ahmed@email.sc.edu (R. U. Ahmed)

\section{To cite this article:}

Sourav Banerjee, Riaz Uddin Ahmed. Phonon Confinement Using Spirally Designed Elastic Resonators in Discrete Continuum. International Journal of Materials Science and Applications. Vol. 3, No. 1, 2014, pp. 6-13. doi: 10.11648/j.ijmsa.20140301.12

\begin{abstract}
Periodic and chiral orientation of microstructures, here we call phononic crystals, have extraordinary capabilities to facilitate the innovative design of new generation metamaterials. Periodic arrangements of phononic crystals are capable of opening portals of non-passing, non-dispersive mechanical waves. Defying conventional design of regular periodicity, in this paper spirally periodic but chiral orientation of resonators are envisioned. Dynamics of the spirally connected resonators and the acoustic wave propagation through the spirally connected multiple local resonators are studied using fundamental physics. In present study the spiral systems with local resonators are assumed to be discrete media immersed in fluid. In this paper it is assumed that acoustic or ultrasonic waves in fluid are propagated along the plane of the spiral resonators and thus only the longitudinal wave mode exists due to nonexistence of shear stress in the fluid. Lagrangian formulation of the spiral systems were employed to obtain the governing Euler-Lagrange equation of the system. Discrete element method was employed to verify the equation assuming nearest neighboring effect.
\end{abstract}

Keywords:Spiral Resonators, Phonon Confinement, Discrete Continuum Model, Spiral Metamaterials

\section{Introduction}

The aim of this paper is to demonstrate the possibility of phonon confinements in a set of spirally connected phononic crystals. In last few years, phononic crystals either in discrete or embedded in the acoustic materials have received significant attention [1-2]. Band gap manipulation is increasingly important in periodic structures to diversify the applications of the novel materials and the phononic crystals Tunabilitiy of frequency band structures in engineered materials are of significant interest due to their many practical applications [3]. Acoustic frequency filtration, vibration isolation, manufacturing of ultrasonic array transducers, improve clarity of acoustic imaging using photon-phonon coupling, micro to nano scale acoustic devices etc. are few applications where frequency manipulations are extremely valuable. Bragg scattering in periodic media is the most important phenomena that is used in both photonics and phononics for creating frequency band gaps. But in photonics there are some possibilities of non-Bragg type band gaps that are called polariton [4] band gaps originates from photon-phonon coupling. Similar phenomenon of phonon-photon coupling can also be found in superlattices. Also recently in phononics, additional frequency band gaps are reported by creating a local resonance effect [2]. In addition to the Bragg scattering, in the periodic structures made of embedded local resonators, negative bulk modulus and negative effective mass causes additional low frequency band gaps [5]. Mass-in-mass unit cells were proposed where the effective mass of the cell becomes negative at frequencies near the local resonance frequency of the resonators due to special decay of the wave amplitudes [6]. Low frequency can be controlled by introducing locally resonant components into the phononic crystals [7]. Based on the previous researches it is envisioned that there is a possibility to open a new portals of low-frequency forbidden bands by arranging the local resonator (phononic crystal) in a certain geometric fashion. Band gaps can also be obtained by altering the geometry of the local resonator [8].

In 2003, Hirsekorn et al. [7] performed a numerical simulation of acoustic wave propagation through sonic crystals consisting of local resonators using the local interaction simulation approach (LISA). It was found that there are three strong attenuation bands at frequencies between 0.3 and $6.0 \mathrm{kHz}$, which does not depend on the periodicity of the crystals. With the Lamb waves, extremely low frequency band gaps (BG) can be obtained by 
periodically depositing single-layer or two-layer stubs on the surface of a thin homogeneous plate [9]. Caballero et al. [10] showed that by placing two-dimensional square and triangular lattices (made of rigid cylinders) in the air, absolute sonic band gaps are created. Such band gapscan be further increased by reducing the structure symmetry or by introducing chirality in the geometry. Based on the idea of localized resonant structures, Liu et al. [11] fabricated sonic crystals that exhibit spectral gaps with a lattice constant two orders of magnitude smaller than the relevant wavelength. Even after such magnificent efforts sonic bands are mostly limited to fewer (one or two) bands gaps with smaller band widths. Superposing the ideas of Liu et al. and Caballero et al. here we envision that geometric configuration of local resonators and there mode of intra-connections could result wider band gaps that would be lucrative for phononic applications and are yet to be explored. Hence, instead of creating stop bands, here we propose spiral system of resonators that are capable of creating selective pass bands by confining the phonons within a desired frequency band.

Naturally obtained spirals (e.g. Cochlea in human ear) shows great advantages in filtering desired frequency of sound waves. Significance of the physics of spiral is evident throughout our nature. Density waves in galaxies, geometrical configuration of the sensory system of the Cephalopodas, geometrical configuration in Cochlea of human ear and numerous botanical species demonstrate the spiral pattern [12]. Appearance of this spiral chirality is sufficiently diverse in nature (e.g. Archimedean, Logarithmic, Golden and Fermat's spiral patterns etc.).Few research can be found in the field of photonics, where guiding of electromagnetic wave by using spiral resonators $[13,14]$ were demonstrated. For most cases Archimedean spiral orientations were used. He et al. [15] proposed a three-dimensional chiral metamaterial consisting of arrays of the multi-layered mutually twisted metallic spirals which can exhibit negative refractive index at terahertz frequencies. Isik et al. [16] determined the electromagnetic response of the particles arranged in Archimedean spiral by using point group symmetry and the methods of crystallography. In 2011, Elford et al. [17] proposed a Bernoulli type spiral coil resonator to attenuate sound pressure level at low frequency region. They found three attenuation (greater than two) areas within the frequency range $0-2.2 \mathrm{kHz}$. They also illustrated that attenuation area can be shifted slightly by changing the number turns in the coil. Then a seashell resonator was also proposed, which is quite similar to naturally obtained nautilus shell that can attenuate sound pressure level at wide range of frequencies.

Inspired by nature, spirally arranged phononic crystals in fluid (air or liquid) is proposed in this study, where cylindrical local resonators are placed both in Archemedian and Logarithmic spiral orientation. To understand the fundamental physics a relatively simple physical model is proposed where all resonators are assumed to be discrete elastic resonators connected via elastic springs (tangential springs). Also to keep the shape of the spiral configuration intact, it is assumed that all the resonators are connected with the central resonator placed at the center of the spiral configuration via another set of elastic springs (radial springs). Shadowing effect of spiral rings are ignored in this derivation, however, to be exact such consideration could be valuable and is under investigation. A parametric study was performed to understand the effect of the spring constants on wave dispersion phenomenon. Mass, radial spring constants and tangential spring constants were varied to see their effect on phonon confinement.

\section{Mathematical Formulation}

\subsection{Euler-Lagrange Equation}

Figure 1 shows the proposed phononic crystal system with spirally connected local resonators. Based on previous studies the local resonators are designed with hard metal coated with soft deformable material e.g. rubber. The proposed system is modeled as a discrete spring mass system. Damping is ignored in the forging derivation. All individual resonators are assumed to be connected with the neighboring resonators on either side, via elastic springs, in series. Simultaneously each resonator is also assumed to be connected with the origin of the spiral configuration, via a different spring to prevent the shape of the spiral. The proposed spring-mass system is shown in Figure 2 (a) and three representative resonators in spiral are depicted in Figure 2(b). As shown in Figure 2(b) each resonator (e.g. $i$-th resonator) is subjected to three forces $F_{i+1}, F_{i} \& F t_{i}$. Displacements of the center of the $i$-th resonator are divided in to two components $x_{i} \& y_{i}$. Similarly the displacements of the neighboring resonators are $x_{i+1}, y_{i+1} \& x_{i-1}, y_{i-1}$ for $i+1$-th and $i-1$-th resonators, respectively. Mass of the $i$ - thresonatoris $m_{i}$. Hence, the Lagrangian [18] of the spiral system can be written in the general form.

$$
L=\frac{1}{2} \sum_{i=1}^{n} m_{i}\left({\dot{x_{l}}}^{2}+\dot{y}_{l}^{2}\right)-\sum_{i=1}^{n} U\left|x_{i}-x_{i-1}\right|\left|y_{i}-y_{i-1}\right|+U\left|x_{i}-x_{i+1}\right|\left|y_{i}-y_{i+1}\right|+U\left|x_{i}-x_{0}\right|\left|y_{i}-y_{0}\right|(1)
$$

Applying the principal of least action we achieve the Euler-Lagrange equation [40] of motion as follows

$$
\frac{\partial L}{\partial x_{i}}-\frac{d}{d t}\left(\frac{\partial L}{\partial \dot{x}_{l}}\right)=0 ; \frac{\partial L}{\partial y_{i}}-\frac{d}{d t}\left(\frac{\partial L}{\partial \dot{y}_{l}}\right)=0
$$




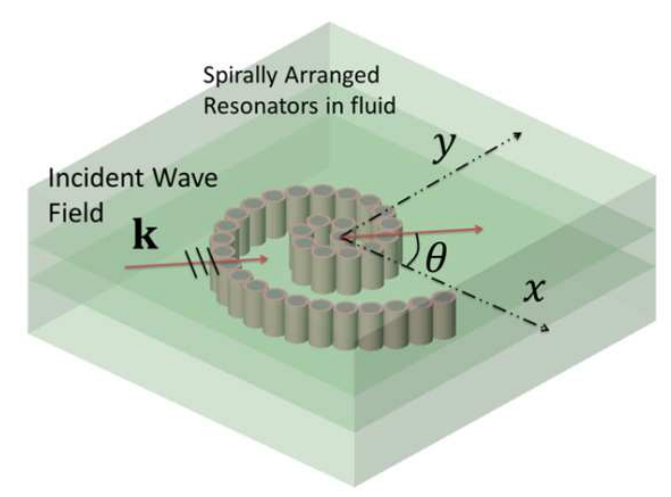

Figure 1. A possible arrangement of phononic crystal in Logarithmic spiral form. Individual resonators are connected to each other through epoxy strings casted together. The system is submerged in fluid to neglect the shear mode or rotation degrees of freedom.

$$
\begin{aligned}
m_{i} \ddot{x}_{\imath}=x_{i} K x x_{i}+y_{i} K x y_{i}+x_{i+1} K x x_{i+1}+x_{i-1} K x x_{i-1}+y_{i+1} K x y_{i+1}+y_{i-1} K x y_{i-1} \\
m_{i} \ddot{y}_{\imath}=x_{i} K y x_{i}+y_{i} K y y_{i}+x_{i+1} K y x_{i+1}+x_{i-1} K y x_{i-1}+y_{i+1} K y y_{i+1}+y_{i-1} K y y_{i-1}
\end{aligned}
$$

where,

$$
\begin{gathered}
K x x_{i}=\left[-K_{i+1}\left(\widehat{n 1}_{i}^{i+1}\right)^{2}-K_{i-1}\left(\widehat{n 1}_{i}^{i-1}\right)^{2}-K t_{i}\left(\widehat{n 1}_{i}^{0}\right)^{2}\right] \\
K x y_{i}=\left[-K_{i+1}\left(\widehat{n 1}_{i}^{i+1}\right)\left(\widehat{n 2}_{i}^{i+1}\right)-K_{i-1}\left(\widehat{n 1}_{i}^{i-1}\right)\left(\widehat{n 2}_{i}^{i-1}\right)-K t_{i}\left(\widehat{n 1}_{i}^{o}\right)\left(\widehat{n 2}_{i}^{o}\right)\right] \\
K x x_{i+1}=\left[K_{i+1}\left(\widehat{n 1}_{i+1}^{i}\right)^{2}\right] K x x_{i-1}=\left[K_{i-1}\left(\widehat{n 1}_{i-1}^{i}\right)^{2}\right] \\
K x y_{i+1}=\left[K_{i+1}\left(\widehat{n 1}_{i+1}^{i}\right)\left(\widehat{n 2}_{i+1}^{i}\right)\right], \\
K x y_{i-1}=\left[K_{i-1}\left(\widehat{n 1}_{i-1}^{i}\right)\left(\widehat{n 2}_{i-1}^{i}\right)\right] \\
K y x_{i}=\left[-K_{i+1}\left(\widehat{n 1}_{i}^{i+1}\right)\left(\widehat{n 2}_{i}^{i+1}\right)-K_{i-1}\left(\widehat{n 1}_{i}^{i-1}\right)\left(\widehat{n 2}_{i}^{i-1}\right)-K t_{i}\left(\widehat{n 1}_{i}^{o}\right)\left(\widehat{n 2}_{i}^{o}\right)\right] \\
K y y_{i}=\left[-K_{i+1}\left(\widehat{n 2}_{i}^{i+1}\right)^{2}-K_{i-1}\left(\widehat{n 2}_{i}^{i-1}\right)^{2}-K t_{i}\left(\widehat{n 2}_{i}^{0}\right)^{2}\right], \\
K y x_{i+1}=\left[K_{i+1}\left(\widehat{n 1}_{i+1}^{i}\right)\left(\widehat{n 2}_{i+1}^{i}\right)\right] K y x_{i-1}=\left[K_{i-1}\left(\widehat{n 1}_{i-1}^{i}\right)\left(\widehat{n 2}_{i-1}^{i}\right)\right], \\
K y y_{i+1}=\left[K_{i+1}\left(\widehat{n 2}_{i+1}^{i}\right)^{2}\right], K x y_{i-1}=\left[K_{i-1}\left(\widehat{n 2}_{i-1}^{i}\right)^{2}\right]
\end{gathered}
$$

Considering $n$ numbers of resonators ( $i$ runs between 1 to $n$ ) in the system, the governing equation of motion can be obtained by arranging the equation (3) and (4) in a matrix form. The generalized dynamic equation for $n$ numbers of resonators in spiral pattern can be written asMä $=\underline{\mathrm{Kq}}$ where, $\underline{M}$ the diagonal mass matrix, $\underline{K}$ the stiffness matrix and $\mathrm{q}=\left\{\begin{array}{lllll}x_{1} & \ldots & x_{n} y_{1} & \ldots & y_{n}\end{array}\right\}^{T}$

\subsection{Eigen Value Solution: Natural Frequencies and Dispersion}

Dynamical behavior of the spiral resonators is studied first. Assuming $x_{i}=A_{i} e^{-j \omega t} \& y_{i}=B_{i} e^{-j \omega t}$, we obtain an eigen value problem from the equation (6) which can be written as $\left[\underline{\mathrm{K}}-\omega^{2} \underline{\mathrm{M}}\right] \lambda=0$

Where, $\boldsymbol{\lambda}=\left\{\begin{array}{lllllll}A_{1} & \ldots & A_{n} & B_{1} & \ldots & B_{n-1} & B_{n}\end{array}\right\}^{T}$ eigen vector representing the mode shapes of the spiral system. The fundamental eigen frequencies are obtained by writing a computer program.

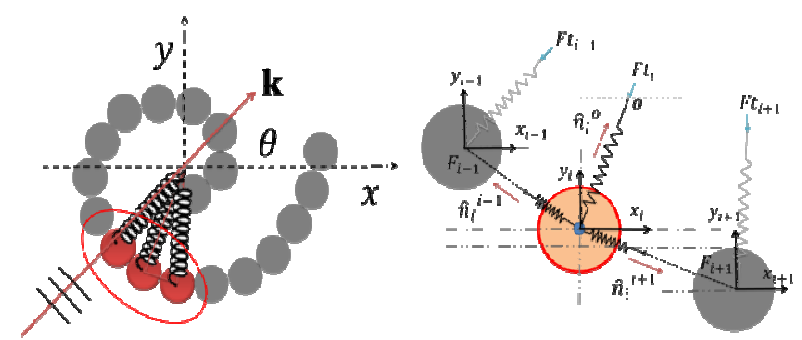

Figure 2.a) Schematic diagram of spirally connected phononic crystals for metamaterials; b) Representative spiral mass spring model using discrete elements

Next the displacement components of the resonators are assumed to have the wave number components in order to discover wave dispersion phenomenon through spirally connected resonators.

Displacements $x_{i}=A_{i} e^{-j \omega t-j \mathrm{k} . \mathrm{r}_{i}} \& y_{i}=B_{i} e^{-j \omega t-j \mathrm{k} . \mathrm{r}_{i}}$ are assumed, where $\mathrm{k}=k_{x} \hat{\imath}+k_{y} \hat{\jmath}$ is the wave number vector and $\mathrm{r}_{i}=x_{i} \hat{\imath}+$ $y_{i} \hat{\jmath}$ is spatial vector measures distance of the $i$-th resonator 
from the origin. Substituting the expressions for the displacements in equations (1) \& (2) and intentionally

dropping the time harmonic term we get

$$
\begin{aligned}
-\omega^{2} m_{i} A_{i}= & A_{i} K x x_{i}+y_{i} K x y_{i}+A_{i+1} e^{\left(j \mathrm{k} . \mathrm{r}_{\mathrm{i}+1}-j \mathrm{k} . \mathrm{r}_{\mathrm{i}}\right)} K x x_{i+1}+A_{i-1} e^{\left(j \mathrm{k} . \mathrm{r}_{\mathrm{i}-1}-j \mathrm{k} \cdot \mathrm{r}_{\mathrm{i}}\right)} K x x_{i-1}+B_{i+1} e^{\left(j \mathrm{k} \cdot \mathrm{r}_{\mathrm{i}+1}-j \mathrm{k} \cdot \mathrm{r}_{\mathrm{i}}\right)} K x y_{i+1} \\
& +B_{i-1} e^{\left(j \mathrm{k} . \mathrm{r}_{\mathrm{i}-1}-j \mathrm{k} \cdot \mathrm{r}_{\mathrm{i}}\right)} K x y_{i-1} \\
-\omega^{2} m_{i} B_{i}= & A_{i} K y x_{i}+y_{i} K y y_{i}+A_{i+1} e^{\left(j \mathrm{k} \cdot \mathrm{r}_{\mathrm{i}+1}-j \mathrm{k} . \mathrm{r}_{\mathrm{i}}\right)} K y x_{i+1}+A_{i-1} e^{\left(j \mathrm{k} \cdot \mathrm{r}_{\mathrm{i}-1}-j \mathrm{k} \cdot \mathrm{r}_{\mathrm{i}}\right)} K y x_{i-1}+B_{i+1} e^{\left(j \mathrm{k} \cdot \mathrm{r}_{\mathrm{i}+1}-j \mathrm{k} \cdot \mathrm{r}_{\mathrm{i}}\right)} K y y_{i+1} \\
& +B_{i-1} e^{\left(j \mathrm{k} \cdot \mathrm{r}_{\mathrm{i}-1}-j \mathrm{k} . \mathrm{r}_{\mathrm{i}}\right)} K y y_{i-1}
\end{aligned}
$$

After arranging all the elements systematically the dispersion equation of the proposed system (spiral resonators) can be written as $\left[\underline{\mathrm{K}}\left(k_{x}, k_{y}\right)-\omega^{2} \underline{\mathrm{M}}\right] \varphi=0$

Where, $\varphi=\left\{\begin{array}{lllllll}A_{1} & \ldots & A_{n} & B_{1} & \ldots & B_{n-1} & B_{n}\end{array}\right\}^{T}$ are theeigen vectors (mode shapes) of the spiral system. The dispersion equations written above can be solved for $\omega$ by assuming digital values of $k_{x} \& k_{y}$. In this paper 2 dimensional wave with wave number $|\mathrm{k}|$ is assumed to propagate along any arbitrary $\theta$ direction with respect to the $x$-axis. $|\mathrm{k}|$ is discretized between 0 to $\pi$ with wave number interval $\pi / 100$. Thus $k_{x}=|\mathrm{k}| \cos (\theta)$ and $k_{y}=|\mathbf{k}| \sin (\theta)$. A computer program was written to solve the dispersionequation and the spiral eigen modes were obtained.

\section{Problem Description}

Solution was obtained from two configurations of the resonators in Archimedean Spiral $(r=5+2 \theta$ and Logarithmic Spiral $\left(r=5 e^{0.2 \theta}\right)$, where dimensions are in $\mathrm{mm}$. The angular distance between two consecutive resonators were $0.4 \pi$. The eigen value problem can be solved for any geometrical configuration of the spirals. Problem can be solved for different material properties of the resonators and materials in which the resonators are placed. For example, individual mass of the resonators, individual spring constants between neighboring resonators and individual spring constants for the springs connecting the resonators to the origin the spiral, can be varied or can be designed as per the requirements. In this paper, the local resonators are assumed to be lead cylinders of mass $5 \mathrm{gm}$ $\left(m_{0}\right)$ and are connected to each other by hard polymer string (source of spring constant $K_{0}=10.5 e 6 \mathrm{~N} / \mathrm{m}$ ). These resonators are then spirally connected with the center of the spiral (source of spring constant $K t_{0}=10.5 e 6 \mathrm{~N} / \mathrm{m}$ ). The complete system is assumed to be submerged in fluid (air or liquid). In this paper three different problems are solved for each type of spirals. Problem 1: Mass of the resonators are assumed to be constant and the stiffness of the springs that connects the neighboring resonators are equal (i.e. $m_{1}=$ $m_{i}=m_{n}=m_{0}, K_{i-1}=K_{i}=K_{i+1}=K_{0}, K t_{i-1}=K t_{i}=$ $\left.K t_{i+1}=K_{0}=K t_{0}\right)$. These assumptions are made for both Archimedean and Logarithmic spirals. Problem 2: Masses of the resonators areincreased by the ratio of their radial distance $\left(m_{i}=m_{0}(r(\theta))\right)$ from the center, keeping spring constants constant. Problem 3: The spring $K t$ that connects the resonators with the center of the spiral (radial springs), are increased by the ratio of the distance of the resonators $\left(K t_{i}=K t_{0}(r(\theta))\right)$ from the center, keeping the mass of the resonators constant. Problem 4: The Kt spring that connects the resonators with the center of the spiral, are decreased by the ratio of the distance of the resonators $\left(K t_{i}=K t_{0}(1 / r(\theta))\right)$ from the center, keeping the mass of the resonators constant. Problem 5: Keeping equal mass and equal radial spring constants, $K_{i}$ is increased by the ratio of their distance from the center $\left(K_{i}=K_{0}(r(\theta))\right)$. Problem 6: Keeping equal mass and equal radial spring constants of the resonators, $K_{i}$ are decreased by the ratio of the distance of the resonators $\left(K_{i}=K_{0}(1 / r(\theta))\right)$ from the center. Problem 7: Keeping the mass of the resonators constants spring constant for $K_{i}$ are considered $1 / 10$ of the spring constant $K t_{i}$ (i.e. $K_{i}=K t_{i} / 10$ ). This assumption is valid, especially when the resonators are coated with softer materials.

\section{Results and Discussions}

Natural frequencies of the Archimedean and Logarithmic spiral systems are shown is Figure 3(a), obtained from Problem 1 and Problem 2. Figure 3(b) shows the deformed or new position of the resonators oriented in Logarithmic spiral obtained from two consecutive vibration modes $\left(26^{\text {th }}\right.$ and $27^{\text {th }}$ eigen vectors) where sudden jump in natural frequencies from $28 \mathrm{KHz}$ to $60 \mathrm{KHz}$ is apparent (from Figure 3(a)). Although sudden jumps are evident from Figure 3(a), such jumps are apparent, if the natural frequencies are plotted keeping track of correlation between the numbed of degrees of freedom and the identities of the resonators in spiral order. Factually no frequency jump in eigen modes were observed from Problem 1 and Problem 2. Maximum natural frequency of the spiral system was found to be around $90 \mathrm{KHz}$. It is observed that $a$ and $b$ parameters in spiral equation $(r=a+b \theta)$ or $\left(r=a e^{b \theta}\right)$ play a key role in obtaining certain natural frequencies (not shown) in their vibrational modes. Similar study was performed in Problem 3 and Problem 4. Radial spring constants were increased (Problem 3) and then decreased (Problem 4) by the ratio of their radial distance and the natural frequencies obtained are plotted in Figure 4 (a). Figure 4(b) shows the natural frequencies of the system when Problem 5 and Problem 6 are solved. From Figure 4(a) it can be seen that there are no obvious gap in the natural frequencies of the system similar to Figure 3(a), however, a narrow band gap in natural frequencies were observed in both Archimedean and Logarithmic spiral systems between $15 \mathrm{KHz}-85 \mathrm{KHz}$ when the spring constants for the springs connecting the resonators to each other are increased or decreased, radially. 

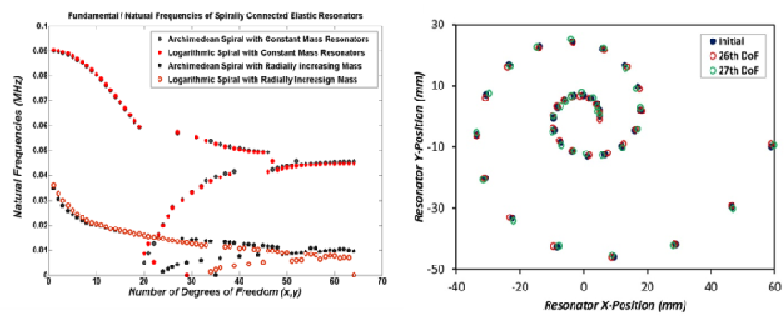

Figure 3.a) Natural vibrational frequencies in Discrete Archemedian and Logarithmic spiral system with constant and radially increasing mass; $b$ ) Displaced position of the centers of the resonators in Logarithmic spiral system for $26^{\text {th }}$ and $27^{\text {th }}$ vibrational mode where a sudden jump is in natural frequency is found.

Next the wave propagation through the proposed spiral system is studied. Here, $e^{i(\mathbf{k} . \mathbf{x}-\omega t)}$ plane wave incident was assumed and the dispersion equation was solved by discretizing the wave numbers to obtain the eigen frequencies. Figure 5 shows the frequency eigen modes within a band $0-\pi$ of the wave numbers obtained from Logarithmic spiral system. In Figure 5(a) marking along the $x$ axis $(0-1,1-2 \& 2-3)$ represents the wave number between $0-\pi$ for the propagation of wave along $0^{0}$, $45^{\circ} \& 90^{\circ}$, respectively. It is apparent that all the possible eigen modes are confined within a specific band of the frequencies. The system is modeled as a discrete system and thus discrete numbers of eigen modes were obtained. All eigen modes were found to be confined within $20 \mathrm{KHz}-100$ $\mathrm{KHz}$ when the mass of the resonators are kept constant (Problem 1). Similar confined band was obtained but shifted between $3 \mathrm{KHz}-25 \mathrm{KHz}$ when the mass of the resonators were increased gradually (Problem 2). Hence, by manipulating the resonator's mass, it is possible to change the governing pass band of the spiral resonator system. Further a closer investigation was carried out. The variation of eigen frequencies, with respect to the wave numbers between $-\pi$ to $\pi$ for the first two modes (wave propagation along $90^{\circ}$ ) are shown in the Figure 5(b). It can be seen that there are many crossover zones between two modes. A closer view of the section marked in blue is shown in Figure 5(c).
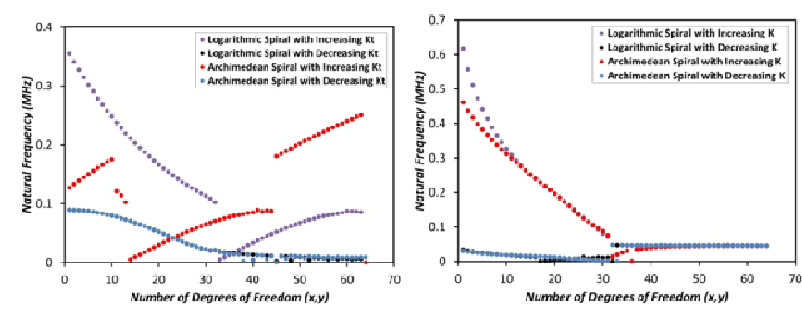

Figure 4.a) Natural vibrational frequencies in Discrete Archemedian and Logarithmic spiral system with constant mass, constant tangential spring constants but radially increasing/decreasing radial spring constants; $b$ ) Natural vibrational frequencies in Discrete Archemedian and Logarithmic spiral system with constant mass, constant radial spring constants but radially increasing/decreasing tangential spring constants.

The radial spring constants are the design parameter of the spiral system and hence a study was conducted to understand their impact on eigen frequencies. Problem 2 and Problem 3 was solved and very complicated mode patterns were observed. Highly dispersive, weakly dispersive and nondispersive wave modes were generated simultaneously. In Figure 6(a) marking along the $x$ axis $(0-1,1-2 \& 2-3)$ represents the wave number between $0-\pi$ for the propagation of wave along $0^{0}, 45^{0} \& 90^{\circ}$, respectively. Completely new finding compared to Problem 1 and 2 was obtained when the radial spring constants are increased radially by the ratio of their radial distance. A definite pass band of frequencies between $35 \mathrm{KHz}-95 \mathrm{KHz}$ was observed along with the nondispersive wave modes between $290 \mathrm{KHz}-355 \mathrm{KHz}$. However, all such modes were found to be dispersive within the wave number band $0-0.1$. Jump between two consecutive wave modes were increasing with the increase in radial spring constant. Between frequencies 0 - $35 \mathrm{KHz}$ no wave modes were found. Indication of modal crossovers (cross-talk) at higher frequencies were found (e.g. marked in red circle in Figure 6(a)) when the wave propagated along the $45^{\circ} \& 90^{\circ}$. Similar crossovers were apparent at lower frequencies (between $100 \mathrm{KHz}-120 \mathrm{KHz}$ ) when the wave propagated along the $x$ axis (propagation angle $0^{0}$ ). These crossovers appeared periodically along the wave number axis and that periodicity was varied with varying frequency. A closer view of the wave modes within in the black box (marked in Figure 6(a)) is shown in Figure 6(b). A mode was found to be symmetric about the wave number 0.5585 , which is essentially the mode associated with $x$ displacement of the 18 th resonator at approximately $30^{\circ}$ angle with the $x$ axis at a distance of $19.481 \mathrm{~mm}$. Dividing the $x$ component of the radial distance by the wave number 0.5585 , it can be seen that approximately 30 full wave form can fit within the radial distance of the 18th resonator. Similar phenomena are evident in many places in the dispersion curves. After solving Problem 4 (the radial spring constants were decreased radially) it was found that the stop band was reduced to $0-8 \mathrm{KHz}$ keeping the upper limit of the mode confinements within $95 \mathrm{KHz}$.
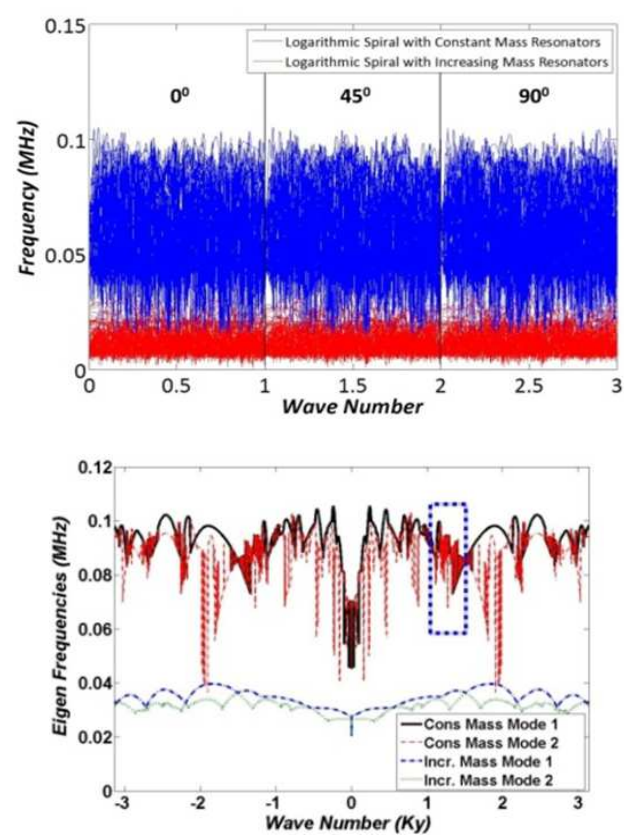


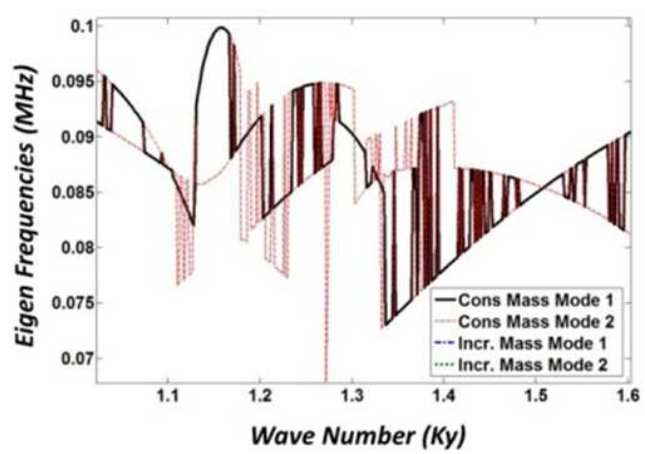

Figure 5.top) Evidence of phonon confinement (all possible real modes) in discrete Logarithmic spiral system with constant and radially increasing mass; middle) Real modes $1^{\text {st }}$ and $2^{\text {nd }}$ modes between wave number $-2 \pi$ to $2 \pi$ in discrete Logarithmic spiral system with constant and radially increasingmass ; bottom) Zoomed view within the blue box : Real modes $I^{s}$ and $2^{\text {nd }}$ modes between wave number $-2 \pi$ to $2 \pi$ in discrete Logarithmic spiral system with constant and radially increasing mass
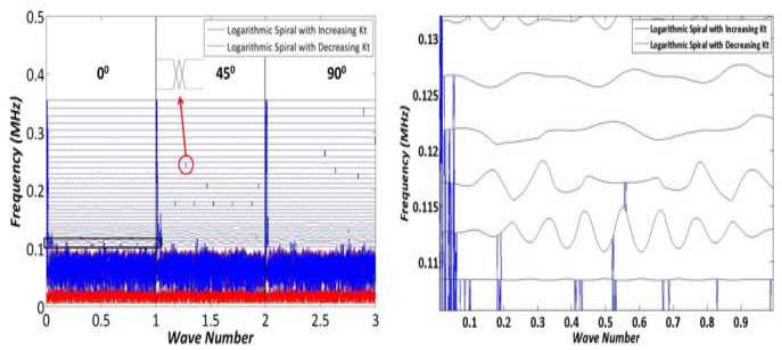

Figure 6.a) Evidence of phonon confinement (all possible real modes nondispersive wave modes were found that results zero group velocity) in discrete Logarithmic spiral system with radially increasing/decreasing radial spring constant; b) Zoomed view within the black box in Figure 6(a)

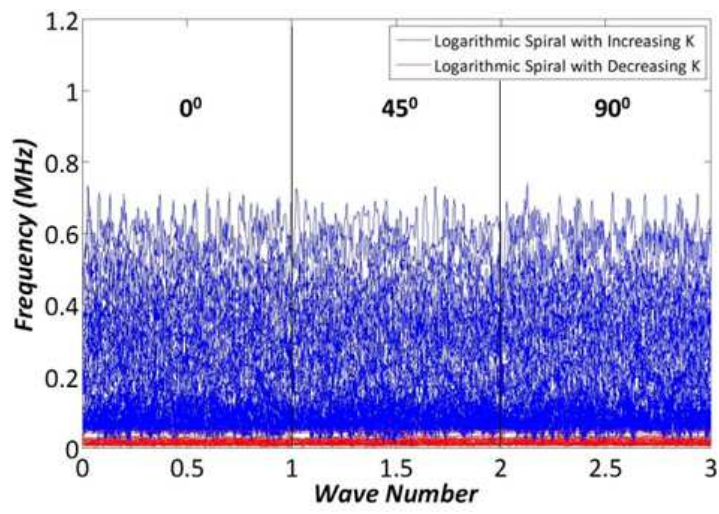

Figure 7.Evidence of phonon confinement (all possible real modes) in discrete Logarithmic spiral system with radially increasing/decreasing tangential spring constant; b) Zoomed view within the black box in Figure $6(a)$

Problem 6 and 7 were studied where the spring constants of the springs connecting two consecutive resonators are increased and decreased, respectively. Figure 7(a) shows the dispersion phenomenon demonstrated by the Problem 6 and Problem 7. When the spring constants $\left(K_{i}\right)$ are increased as per the problem description in Problem 6 the wave confinement were found to be within $20 \mathrm{KHz}$ to $700 \mathrm{KHz}$. Hence, below $20 \mathrm{KHz}$ no wave modes were found. Similarly, when the spring constants are decreased by the ratio of the radial distance of the resonators, wave confinement were separated in to two zones, zone 1, is wider band between 5 $\mathrm{KHz}-25 \mathrm{KHz}$ and zone 2, a narrow band between $45 \mathrm{KHz}-$ $47 \mathrm{KHz}$. Multiple modal crossover points between these two zones were identified similar to the points shown in Figure 6 (a). Hence between $25 \mathrm{KHz}-45 \mathrm{KHz}$, no modes were found along the wave number axis except at the point of crossovers.

Another interesting phenomenon that was observed, after solving the Problem 7, is shown in Figure 8. Figure 8(a) shows the natural frequencies of the spiral system obtained when the tangential springs constants are ten time less than the radial spring constants. Such parametric variation created a band of frequencies which are not possible to be the natural frequency of the spiral system and then almost half of the wave modes found to be nondispersive. Next, wave propagation through the Logarithmic spiral system was studied (wave propagating along $\mathrm{x}$ direction (see Figure 8(b) \& (c))). A clear band gap at lower frequencies (similar to the above examples) was observed and another band gap between $30 \mathrm{KHz}-45 \mathrm{KHz}$ was found. Just above this band gap another narrow pass band between $46 \mathrm{KHz}-46.6 \mathrm{KHz}$ was observed. The band gap between $30 \mathrm{KHz}-45 \mathrm{KHz}$ were found to be dependent on the ratio of tangential and radial spring constants. Hence, softer springs between resonators along the tangential direction are capable of creating larger band gaps at lower frequencies. Modal crossover points every $2 \pi$ interval were evident as discussed before. These modal crossovers act as bridge between two pass bands at certain wave number zone. Hence, there are significant possibilities of manipulating these behaviors for practical use in frequency filtration.
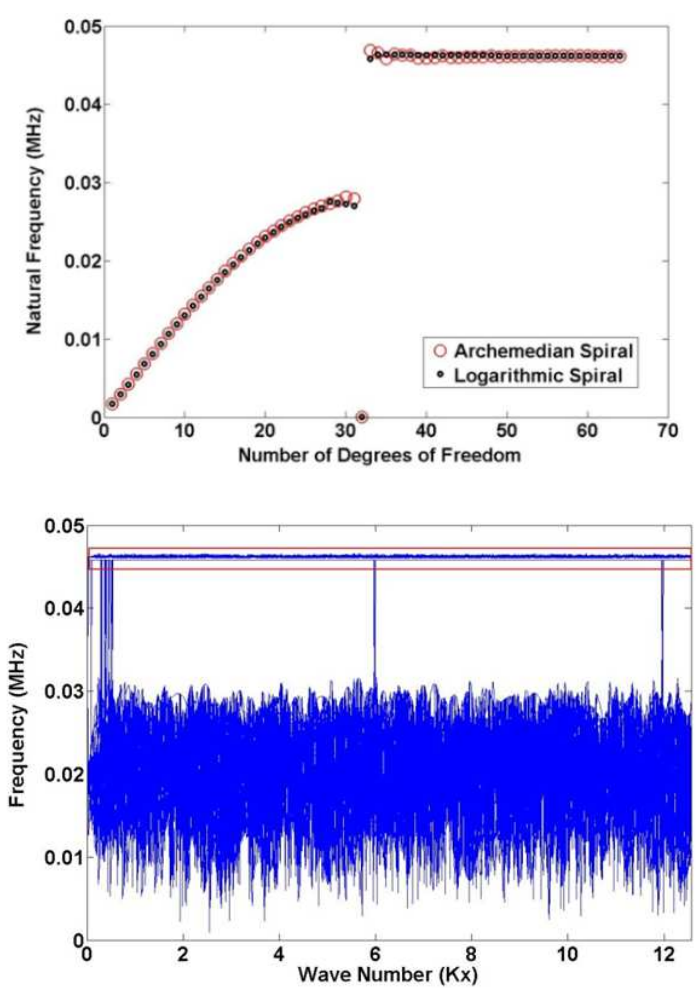


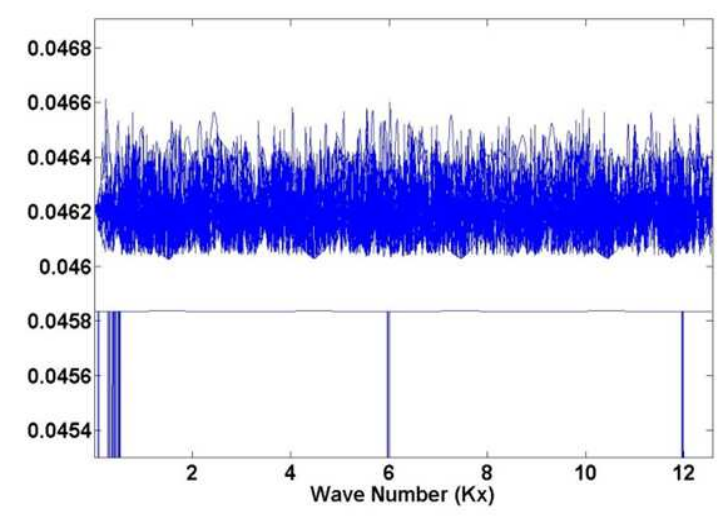

Figure 8.a) Natural vibrational frequencies in Discrete Archemedian and Logarithmic spiral system when tangential spring constants are 10 times less than the radial spring constants. b) Wave dispersion through the system showing existence of two pass bands between a stop band c) zoomed view of the modes within the red window in Figure 8(b)

\section{Conclusion}

From the above study the following comments can be made. Spiral modal behavior is geometry dependent. Archimedean and Logarithmic Spirals have similar phenomena but different frequency responses.Inthe vibration analysis of the Logarithmic spirals, the nonexistence of the natural frequencies is correlated with the frequency band gaps that are obtained when the wave propagation through the spiral system was studied. Increasing spring constants increased the frequencies of the passing band of the wave modes, whereas increased mass $\left(m_{i}\right)$ decreased the frequency bands of the wave modes. Different spring constants for radial springs $\left(K t_{i}\right)$ and tangential springs $\left(K_{i}\right)$ could potentially result in separation of band structures with multiple pass bands. However, it is affirmative from the results that low frequency non passing zone can be created and all the possible wave modes can be confined within certain band of frequencies. Suitable selection of material for the resonators and materials for the springs will provide extreme design flexibility for frequency control and wave guiding.

\section{Acknowledgements}

The project is partially funded by Office of Vice President of Research and Department of Mechanical Engineering at the University of South Carolina.

\section{References}

[1] Montero de Espinoza, F.R., Jimenez, E., Torres, M., (1998). Ultrasonic Band Gap in a Periodic Two-Dimensional Composite. Phys. Rev. Lett. 80, 1208

[2] Li, X., Liu, Z.Y., (2005). Coupling of cavity modes and guiding modes in two-dimensional phononic crystals. Solid State Commun. 133, 397.

[3] Poulton, C. G., Movchan, A. B., McPhedran, R. C., Nicorovici, N. A., and Antipov, Y. A., (2000). Eigenvalue
Problems for Doubly Periodic Elastic Structures and Phononic Band Gaps. Proc. R. Soc. London, Ser. A, 456, 2543-2559.

[4] Xu, Y. L., Chen, C. Q., Tian, X. G., (2013). Phonon-polarization and band structure of electro-magneto-acoustic $\mathrm{SH}$ wave propagation oblique to the periodic layers piezoelectric. Physics Letters A., 377, 895-902.

[5] Li, J., and Chan, C. T., (2004). Double-Negative Acoustic Metamaterial. Phys. Rev. E, 70, 055602.

[6] Yao, S. S., Zhou, X. M., and Hu, G. K., (2008). Experimental Study on Negative Effective Mass in a 1D Mass-Spring System. New J. Phys., 10, 043020

[7] Hirsekorn, M., Delsanto, P.P., Batra, N. K., Matic, P., (2004). Modelling and simulation of acoustic wave propagation in locally resonant sonic materials. Ultrasonics, 42(19), 231-235.

[8] Hsu, J. C., (2011). Local resonances-induced low-frequency band gaps in two-dimensional phononic crystal slabs with periodic stepped resonators. J. Phys. D: Appl. Phys., 44, 055401 .

[9] Caballero, D., S'anchez-Dehesa, J., Rubio, C., M'artinez-Sala, R., S'anchez-P'erez, J., V., Meseguer, F., Llinares, J., (1999). Large two-dimensional sonic band gaps. Phys. Rev. E, 60(6), R6316-R6319.

[10] Caballero, D., S'anchez-Dehesa, J., Rubio, C., M'artinez-Sala, R., S'anchez-P'erez, J., V., Meseguer, F., Llinares, J., (1999). Large two-dimensional sonic band gaps. Phys. Rev. E, 60(6), R6316-R6319.

[11] Liu, Z., Chan, C. T., and Sheng, P., (2005). Analytic Model of Phononic Crystals WithLocal Resonances. Phys. Rev. B, 71, 014103.

[12] Mainzer, K., Symmetries of Nature: A Handbook for Philosophy of Nature and Science, Walter de Gruyter\& Co., ISBN 3-11-012990-6, 1998.

[13] Nemer, S., Sauviac, B., Bayard, B., Nader, C., Bechara, J., and Khoury, A., (2011). Modelling resonance frequencies of a multi-turn spiral for metamaterial applications. Progress In Electromagnetics Research C, 20, 31-42.

[14] Baena, J., Marqués, R., Medina, F., \& Martel, J., (2004). Artificial magnetic metamaterial design by using spiral resonators. Physical Review B, 69(1), 1-5.

[15] He, M., Han, J., Tian, Z., Gu, J., Xing, Q., (2011). Negative refractive index in chiral spiral metamaterials at terahertz frequencies. Optik - International Journal for Light and Electron Optics, 122(18), 1676-1679.

[16] Isik, O., Esselle, K., P., (2009). Analysis of spiral metamaterials by use of group theory. Metamaterials, 3(1), 33-43. 
[17] Elford, D., P., Chalmers, L., Kusmartsev, F., V., Shallowe, G., M., (2010). Acoustic band gap formation in metamaterials. International Journal of Modern Physics B, 24 (25-26),
$4935-4945$.

[18] Goldstein, H., Poole, P. Charles, Safko, J., Classical Mechanics, Pearson, ISBN 978-81-317-5891-5, 2011. 